\title{
Memorial consequences of imagination in children and adults
}

\author{
AlP Aslan ANd Karl-Heinz BäUML \\ Regensburg University, Regensburg, Germany
}

\begin{abstract}
Recent work with adults suggests that imagination can impair later recall of previously encoded events but can improve recall of subsequently encoded events. The present study examined the memorial consequences of imagination in children. Kindergartners, first and fourth graders, and young adults studied two successively presented lists of items. Between the two lists, participants were given an imagination task supposed to create a change in mental context. As expected, in adults, the imagination task impaired recall of the previously encoded material (List 1) and improved recall of the subsequently encoded material (List 2). In children, significant List 1 impairment was present from first grade on, but even fourth graders failed to show improvement for List 2 . The results challenge a purely context-based explanation of the memorial costs and benefits of imagination. Instead, they suggest that the two effects are mediated by different mechanisms with different developmental trajectories.
\end{abstract}

Teachers often complain about young children's absentmindedness during lessons. Indeed, in many situations at school, children may mentally drift away from the task at hand and engage in task-irrelevant thoughts and imagination. Leaving alone the fact that such behavior may make the teacher livid, recent research with adults suggests that imagination can have substantial effects on episodic memory (Klein, Shiffrin, \& Criss, 2007; Pastötter \& Bäuml, 2007; Sahakyan \& Delaney, 2003; Sahakyan \& Kelley, 2002). The present study is the first to examine this issue in children.

In their original study on the effect of imagination on episodic memory, Sahakyan and Kelley (2002) had participants learn two lists of unrelated items. After presentation of the first list, participants were given an imagination task: to mentally walk through their parents' home or to describe what they would do if they were invisible. After study of the second list and a retention interval, a recall test for the two lists was conducted. Relative to a no-imagination control condition, the imagination task impaired recall of List 1 (the costs of imagination), but improved recall of List 2 (the benefits of imagination). Imagination was thus found to have not only a detrimental but also a beneficial effect on memory performance, at least in young adults.

It has been suggested that the memorial consequences of imagination reflect a variant of context-dependent memory (Sahakyan \& Kelley, 2002). The imagination task is assumed to cause a more than normal change in participants' mental context, so that the previously encoded material (List 1) and the subsequently encoded material (List 2) become connected to different contextual cues. In contrast, participants in the no-imagination condition are more likely to treat the two sets of materials as parts of the same event and thus connect them to the same mental context cue. The costs of imagination arise because, regarding List 1, the mismatch between the retrieval context and the learning context is larger in the imagination than in the no-imagination condition (Tulving \& Thomson, 1973; for reviews of context-dependent memory, see Eich, 1989, or Smith \& Vela, 2001). The benefits of imagination can be explained in terms of the same (context-change) mechanism. The context change should reduce proactive interference as a result of learning the two lists in different mental contexts and thus improve recall of List 2 (Sahakyan \& Kelley, 2002).

More recent work, however, has indicated that the benefits of imagination may reflect a different, more strategic process. In fact, Sahakyan and Delaney (2003) reported evidence that participants in the imagination condition more often change their encoding strategy from more shallow processing of the previously encoded material (List 1) to deeper processing of the subsequently encoded material (List 2). Participants apparently use the change in mental context to evaluate prior learning and, in an attempt to improve memory for subsequent material, adopt a deeper encoding strategy for the second list. Consistently, if one controls subjects' encoding strategies for the two lists, the benefits of imagination can be eliminated, while the costs remain unaffected (Sahakyan \& Delaney, 2003). Thus, in opposition to the purely context-based account, the memorial costs and benefits of imagination may arise from different mechanisms, the costs from an encoding/retrieval mismatch in contextual cues, and the benefits from a person's deliberate shift to a superior encoding strategy. 
The aim of the present study was to investigate the memorial consequences of imagination in children. Although the pure context account predicts that the costs and benefits of imagination should show the same developmental trajectory, on the basis of Sahakyan and Delaney's (2003) two-factor account, the two opposing memorial effects may show very different developmental trajectories. Indeed, if the benefits of imagination depend on one's deliberate change of encoding strategy on the basis of a metamemorial evaluation of one's own memory performance, then the benefits of imagination may be expected to emerge relatively late in development. It is well known that children show deficits in metamemorial capabilities, some extending even into adolescence (Schneider \& Pressley, 1997). In contrast, children as young as kindergartners are sensitive to contextual changes and show forgetting when the study context mismatches the retrieval context (Bartlett, Burleson, \& Santrock, 1982; Wilkinson, 1988). Although the effects of mental context changes have not been examined yet, these findings suggest that the costs of imagination may emerge at a relatively early stage in development.

In the present experiment, kindergartners, first and fourth graders, and young adults studied two lists of items in preparation for a memory test. Between the two lists, participants were provided an imagination task in which they were asked to describe what they would do if they were invisible (Sahakyan \& Kelley, 2002). After study of the second list and a retention interval, subjects were asked to recall all previously studied items. Replicating prior work, we expected that the imagination task would impair recall of List 1 and improve recall of List 2 in adults. If the costs and benefits of imagination emerged from the same (context-change) mechanism, then the two effects should arise at a relatively early stage in development and show the same developmental trajectory. Alternatively, on the basis of the more recent two-factor account of the memorial consequences of imagination and previous findings on memory development in children, the memorial costs and benefits of imagination should show different developmental trajectories, with the costs arising earlier in development than the benefits.

\section{METHOD}

\section{Participants}

A total of 169 participants from four age groups (kindergartners, first graders, fourth graders, and adults) took part in the experiment. From this original sample, 5 kindergartners were excluded because they were not familiar with the concept of invisibility and thus could not follow task instruction. Further, 2 kindergartners, 1 fourth grader, and 1 adult were excluded because they failed to engage in the imagination task for at least half of the provided time. This yielded a final sample of forty 4 - and 5-year-old kindergartners $(M=4.5, S D=0.5 ; 18$ female, 22 male $)$, forty 6 - and 7-yearold first graders $(M=6.7, S D=0.5 ; 24$ female, 16 male $)$, forty 9- and 10-year-old fourth graders $(M=9.4, S D=0.5 ; 22$ female, 18 male), and 40 young adults $(M=24.6, S D=3.6 ; 23$ female, 17 male). The children were recruited from several kindergartens and elementary schools near Regensburg, Germany, and received a small gift for their participation. The adults were students of Regensburg University and received course credit. All participants were tested individually.

\section{Materials}

Four study lists were constructed, each consisting of six unrelated nouns from word norms for children (Hasselhorn, Jaspers, \& Hernando, 1990; Posnansky, 1978).

\section{Design and Procedure}

The experiment had a mixed design with the between-participants factor of age group (kindergartners, first graders, fourth graders, adults) and the within-participants factors of condition (imagination, no imagination) and list position (List 1, List 2). For each participant, the experiment consisted of two parts that differed in which list had to be learned and which condition was provided. In each part, the items of the first list were presented successively and in random order at a rate of $3 \mathrm{sec}$ per word. After study of the list, participants in the imagination condition were engaged in an imagination task for approximately $60 \mathrm{sec}$. They were asked to imagine being invisible and to report what they would do if they had no responsibility for their actions (Sahakyan \& Kelley, 2002). Participants in the no-imagination condition were engaged in slight conversation for an equivalent amount of time. The conversation was intended to prevent participants from rehearsal of List 1 items and consisted of simple questions such as how old the participant was or how many siblings he/she had. Thereafter, the second list was presented, and, after a 90 -sec distractor task, a recall test for all of the previously studied items was conducted. Half of the participants were asked to recall List 1 items first, and half were asked to recall List 2 items first. Participants had $1 \mathrm{~min}$ per list but were given extra time when needed. The verbal responses were noted by the experimenter. The two parts of the experiment were separated by a 3-min break. The order of the imagination and the no-imagination conditions was counterbalanced across participants, as was the assignment of lists to condition and list position.

\section{RESULTS}

Following the procedures of prior work (e.g., Sahakyan \& Delaney, 2003; Sahakyan \& Kelley, 2002), we analyzed List 1 and List 2 recall separately. An item was counted as correctly recalled if it was named at any time during the test phase, provided that it was part of the current condition's lists. Preliminary analyses revealed that counterbalancing did not influence the overall pattern of results. Neither testing order (List 1 tested first vs. List 2 tested first) nor condition order (imagination condition first vs. no-imagination condition first) showed a main effect, nor did the counterbalancing factors interact with any of the other variables (all $p \mathrm{~s}>.15$ ). Thus, for the analyses reported below, the data were collapsed across counterbalancing conditions. The results for the four age groups are depicted in Figure 1.

Regarding List 1 performance, a $2 \times 4$ ANOVA with the factors of condition (imagination, no imagination) and age group (kindergartners, first graders, fourth graders, adults) revealed a significant main effect of condition $\left[F(1,156)=19.1, M S_{\mathrm{e}}=0.032, p<.001\right]$ and a significant main effect of age group $\left[F(3,156)=90.0, M S_{\mathrm{e}}=\right.$ $0.050, p<.001]$. These main effects reflect higher overall recall in older than in younger participants and reduced recall in the imagination condition in comparison with the no-imagination condition. There was also a significant interaction between the two factors $[F(3,156)=2.8$, $\left.M S_{\mathrm{e}}=0.032, p<.05\right]$, indicating that the effect of the imagination task differed across age groups. Single comparisons revealed that the imagination task impaired List 1 recall in first graders, fourth graders, and young adults 

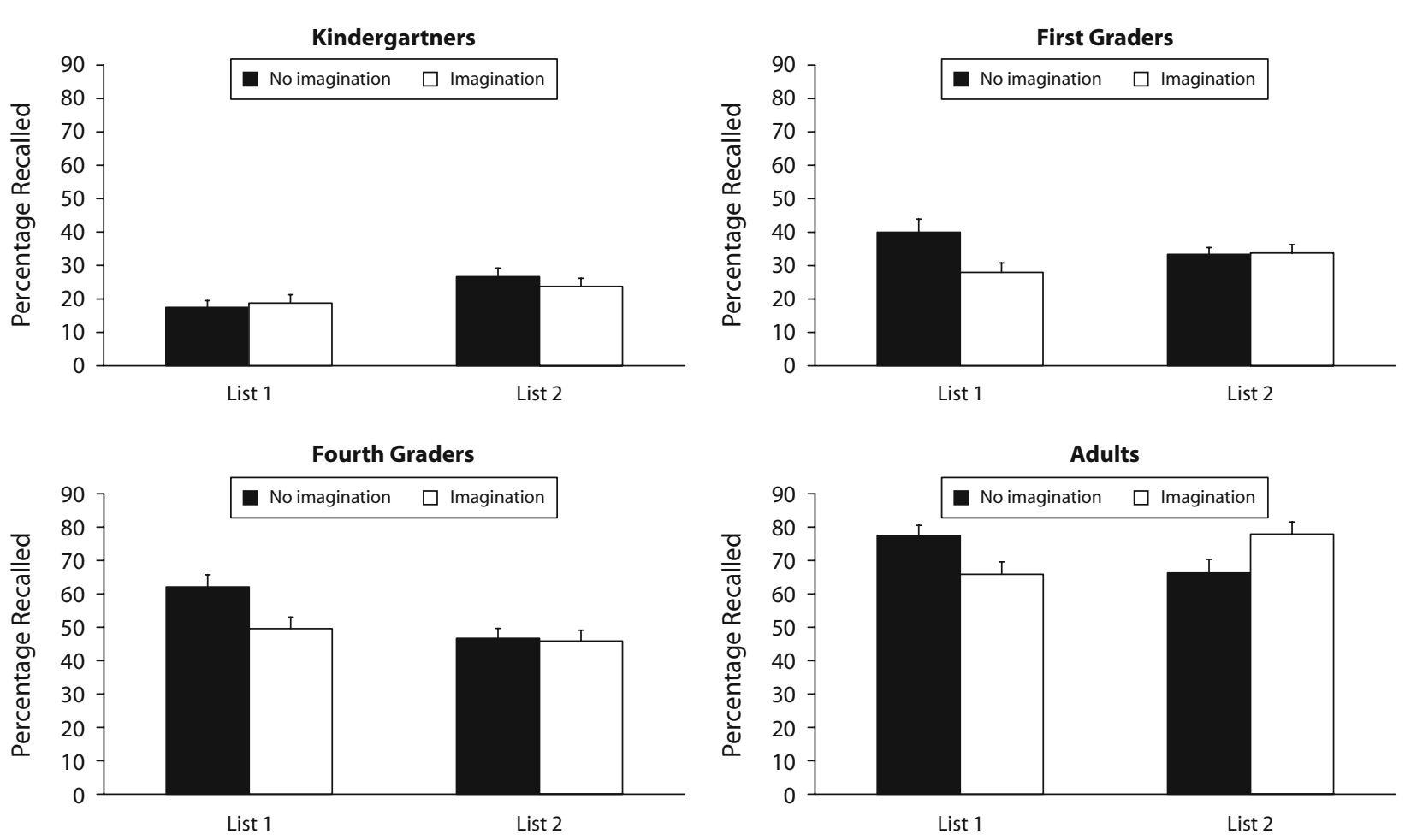

Figure 1. Recall performance in the four age groups as a function of condition (imagination, no imagination) and list position (List 1, List 2). The error bars represent standard errors.

(all $p \mathrm{~s}<.05)$ but did not impair recall in kindergartners $[F(1,39)<1]$.

Regarding List 2 performance, a $2 \times 4$ ANOVA with the factors of condition (imagination, no imagination) and age group (kindergartners, first graders, fourth graders, adults) revealed no main effect of condition $[F(1,156)=$ $\left.1.4, M S_{\mathrm{e}}=0.026, p>.20\right]$ but a significant main effect of age group $\left[F(3,156)=72.2, M S_{\mathrm{e}}=0.046, p<.001\right]$, again reflecting the expected higher overall recall in older than in younger participants. Importantly, there was a significant interaction between the two factors $[F(3,156)=$ 3.3, $\left.M S_{\mathrm{e}}=0.026, p<.05\right]$, reflecting the fact that the imagination task improved List 2 recall only in the adult group $\left[F(1,39)=11.9, M S_{\mathrm{e}}=0.023, p=.001\right]$ but did not improve recall in any of the three children groups [all $\left.F_{\mathrm{s}}(1,39)<1\right]$.

The preceding analysis of List 1 and List 2 recall indicates that the costs of imagination develop sometime between kindergarten and first grade, whereas the benefits develop after fourth grade, suggesting that the two effects follow different developmental trajectories. This suggestion is confirmed by a 2 (condition) $\times 4$ (age group) $\times 2$ (list position) ANOVA, which revealed a significant threeway interaction $\left[F(3,156)=4.1, M S_{\mathrm{e}}=0.032, p<.01\right]$.

\section{Analysis of Level Differences}

To examine whether differences in the four age groups' overall recall level affected the results in the present study, we conducted a series of additional analyses. We first split each age group into high- and low-performing subgroups, on the basis of participants' overall recall level. Although recall level was of course higher in the former group, in none of the four age groups did high- and low-performing participants differ with respect to either the costs or the benefits of imagination (all $p \mathrm{~s}>.30$ ).

In the next step, we directly compared the amount of List 1 forgetting in high-performing kindergartners and low-performing first graders. Although differing in age, the two groups did not differ in overall recall level (both $27.1 \%)$. List 1 forgetting differed significantly between the two groups $(p<.05)$ and was present only in the (lowperforming) first graders $(12.5 \%, p<.05)$, but not the (high-performing) kindergartners $(-3.3 \%, p>.20)$.

In the final step, we directly compared the amount of List 2 improvement in high-performing fourth graders with that of low-performing adults. Though differing in age, the two groups did not differ in overall recall level, with the high-performing fourth graders being even slightly better than the low-performing adults $(61.0 \% \mathrm{vs}$. $56.9 \%)$. Still, List 2 improvement differed significantly between the two groups $(p<.05)$ and was present only in the (low-performing) adults $(15.0 \%, p<.05)$, not in the (high-performing) fourth graders $(-5.0 \%, p>.30)$. Together, these analyses indicate that the suggested developmental trajectories do not depend on the four age groups' overall recall level.

\section{Analysis of Intrusion Errors}

We also analyzed cross-list intrusion errors (i.e., recall of List 2 items when List 1 was tested, and vice versa). The 
overall intrusion rates were relatively low and, within each age group, did not differ across conditions (all $p s>.25$ ). A one-way ANOVA on mean intrusion rates, however, revealed a main effect of age group $(p<.05)$, reflecting the fact that adults showed fewer intrusion errors $(2.5 \%)$ than did the three children groups (kindergartners, 4.7\%; first graders, $5.9 \%$; fourth graders, $5.2 \%$ ). The children groups did not differ in intrusion rates $(p>.50)$.

\section{DISCUSSION}

We replicated prior work by showing that changing an adult's mental context by means of an imagination task can lead to impaired memory for the previously encoded material but can lead to improved memory for the subsequently encoded material (Pastötter \& Bäuml, 2007; Sahakyan \& Kelley, 2002). Going beyond this prior work, we found that the memorial effects of imagination in children differed from those in adults and varied with the children's age. Whereas in kindergartners, imagination induced neither memorial benefits nor memorial costs, first and fourth graders showed the costs but did not show any benefits. These findings challenge a purely context-based explanation of the memorial consequences of imagination, according to which the costs and benefits of imagination should go hand in hand for each age group. Rather, the findings suggest different developmental trajectories for the two memorial effects of imagination and indicate that the costs of imagination arise earlier in development than do the benefits.

The finding that the benefits of imagination arise at a relatively late stage in development is in line with the literature on metamemory development. Metamemory refers to both declarative and procedural knowledge that people have about memory (see, e.g., Flavell \& Wellman, 1977), including knowledge about the efficient use of memory strategies and processes such as the monitoring and regulation of the ongoing memorial activity. Metamemorial skills are one of the last capabilities to develop in memory, with much of the developmental progress occurring during adolescence, and even later (Schneider \& Pressley, 1997). Because the benefits of imagination seem to presuppose such metamemorial capabilities (Sahakyan \& Delaney, 2003), the present results are consistent with the literature on metamemory development.

The present finding that, in contrast to the benefits of imagination, the costs of imagination are present at a relatively early stage in development shows similarities with related work on children's context-dependent memory. Examining the effects of an internal context change, Bartlett et al. (1982), for instance, manipulated children's emotional mood (happy vs. sad) between study and test. They found significant mood-dependent forgetting in 5and 8-year-old children. Manipulating the environmental context between study and test, Wilkinson (1988) obtained similar results in even younger children. The present results agree with this previous work by indicating that the costs of a context change are present from first grade on. Besides, they suggest that, at least in kindergartners, the costs of imagination may be smaller than those of other forms of context changes.

The question arises of whether our failure to find the costs of imagination in kindergartners is a "real" age-related null effect or, alternatively, reflects specifics of our particular experimental setup. Several arguments speak against the latter interpretation. First, we were careful to determine whether children were able to follow task instruction and we excluded those who were not familiar with the concept of invisibility. We also excluded participants who did not engage in the imagination task for a specified amount of time. Second, we examined whether the low recall levels might have obscured potential costs of imagination in kindergartners. As it turned out, neither the low- nor the highperforming kindergartners showed the costs of imagination. In contrast, there was a significant difference in costs between high-performing kindergartners (who did not show forgetting) and low-performing first graders (who showed forgetting), despite the fact that the two groups matched exactly in recall level. Thus, the absence of the costs of imagination in kindergartners seems to be a "real" age-related null effect and not simply a consequence of low recall levels or difficulties with task instruction.

An intriguing feature in the present data is that-except among the kindergartners - recall in the no-imagination condition was characterized by list primacy (i.e., better recall of List 1 than of List 2), which tended to change to list recency (i.e., better recall of List 2 than of List 1) in the imagination condition. Kindergartners, in contrast, exhibited list recency in both conditions. Thus, one might like to argue that, for the youngest children, the slight conversation between the learning of the two lists in the no-imagination condition might have acted as a mental context change similar to the imagination task in the imagination condition. However, results from related paradigms show that young children often exhibit list recency, even in conditions in which two lists are studied in immediate succession without an intervening task (Harnishfeger \& Pope, 1996). This finding suggests that the kindergartners' list recency observed in the present study was not specific to the conversation (or to the imagination task) that was introduced between the two lists.

The present results impose restrictions on theoretical accounts of the memorial consequences of imagination. In line with recent work in adults (Sahakyan \& Delaney, 2003), they challenge a purely context-based explanation of the two effects of imagination. Because this account attributes the two effects to the same (context-change) mechanism, individuals should show the benefits whenever they show the costs of imagination, and vice versa. Although this account can explain the presence of the two effects in adults and the absence of the two effects in kindergartners, it cannot explain why the first and fourth graders showed the costs of imagination, but did not show any benefits. Rather, the observed developmental dissociation between benefits and costs is in line with the proposal that the costs of imagination reflect context-dependent forgetting, whereas the benefits reflect an individual's deliberate shift in strategy from more shallow encoding of previous 
events to deeper encoding of subsequent events (Sahakyan \& Delaney, 2003).

The result that elementary school children reveal only the costs but do not show any memorial benefits of imagination may arrive as a rather awkward message for teachers and other practitioners. Fortunately, however, the costs of context changes appear to be restricted largely to recall situations and to be negligible in recognition tests (Godden \& Baddeley, 1980; Smith, Glenberg, \& Bjork, 1978). Moreover, mentally reinstating the original context can also reduce the costs of imagination (Sahakyan \& Kelley, 2002), leaving us with the picture that imagination itself may remove the costs that it created.

\section{AUTHOR NOTE}

The present research was supported by a grant from the Deutsche Forschungsgemeinschaft (DFG) to K.-H.B. and A.A. (BA 1382/8-1). We thank V. Bauer, B. Burges, B. Diem, T. Dolzer, and A. Fenk for their help with data collection. We also thank R. Bjork, B. Storm, and one anonymous referee for comments on a previous version of the manuscript. Correspondence concerning this article should be addressed to A. Aslan, Department of Experimental Psychology, Regensburg University, 93040 Regensburg, Germany (e-mail: alp.aslan@psychologie uni-regensburg.de)

\section{REFERENCES}

Bartlett, J. C., Burleson, G., \& Santrock, J. W. (1982). Emotional mood and memory in young children. Journal of Experimental Child Psychology, 34, 59-76.

EICH, E. (1989). Theoretical issues in state dependent memory. In H. L. Roediger III \& F. I. M. Craik (Eds.), Varieties of memory and consciousness: Essays in honour of Endel Tulving (pp. 331-354). Hillsdale, NJ: Erlbaum.

Flavell, J. H., \& Wellman, H. M. (1977). Metamemory. In R. V. Kail $\& \mathrm{~J}$. W. Hagen (Eds.), Perspectives on the development of memory and cognition (pp. 3-33). Hillsdale, NJ: Erlbaum.

GodDEn, D., \& BADDEley, A. (1980). When does context influence recognition memory? British Journal of Psychology, 71, 99-104.
Harnishfeger, K. K., \& Pope, R. S. (1996). Intending to forget: The development of cognitive inhibition in directed forgetting. Journal of Experimental Child Psychology, 62, 292-315.

Hasselhorn, M., Jaspers, A., \& Hernando, M.-D. (1990). Typizitätsnormen zu zehn Kategorien von der Vorschule bis zur vierten Grundschulklasse [Typicality norms for ten categories from preschool to fourth grade]. Sprache \& Kognition, 9, 92-108.

Klein, K. A., Shiffrin, R. M., \& Criss, A. H. (2007). Putting context in context. In J. S. Nairne (Ed.), The foundations of remembering: Essays in honor of Henry L. Roediger III (pp. 171-189). New York: Psychology Press.

PAstötter, B., \& BäUmL, K.-H. (2007). The crucial role of postcue encoding in directed forgetting and context-dependent forgetting. Journal of Experimental Psychology: Learning, Memory, \& Cognition, 33, 977-982.

PosnansKy, C. J. (1978). Category norms for verbal items in 25 categories for children in Grades 2-6. Behavior Research Methods \& Instrumentation, 10, 819-832.

SahaKyan, L., \& Delaney, P. F. (2003). Can encoding differences explain the benefits of directed forgetting in the list method paradigm? Journal of Memory \& Language, 48, 195-206.

SahaKyan, L., \& Kelley, C. M. (2002). A contextual change account of the directed forgetting effect. Journal of Experimental Psychology: Learning, Memory, \& Cognition, 28, 1064-1072.

SCHNeIDER, W., \& Pressley, M. (1997). Memory development between two and twenty (2nd ed.). Mahwah, NJ: Erlbaum.

Smith, S. M., Glenberg, A., \& BJork, R. A. (1978). Environmental context and human memory. Memory \& Cognition, 6, 342-353.

Smith, S. M., \& Vela, E. (2001). Environmental context-dependent memory: A review and meta-analysis. Psychonomic Bulletin \& Review, 8, 203-220.

Tulving, E., \& Thomson, D. M. (1973). Encoding specificity and retrieval processes in episodic memory. Psychological Review, 80, 352-373.

WiLKINSON, J. (1988). Context effects in children's event memory. In M. M. Gruneberg, P. E. Morris, \& R. N. Sykes (Eds.), Practical aspects of memory: Current research and issues. Vol. 1: Memory in every day life (pp. 107-111). Chichester, U.K.: Wiley.

(Manuscript received November 22, 2007; revision accepted for publication February 1, 2008.) 